$$
L\left\{v_{r}\right\}=\frac{1}{p}-\frac{\left[\left(1+p^{2} / a^{2}\right)^{1 / 2}-p / a\right]^{2 r-1}}{p\left[1+\left(1+p^{2} / a^{2}\right)^{1 / 2}\right]} . \quad r=1,2, \cdots,
$$

where $a=2(L C)^{-1 / 2}$.

It follows from (12) that

$$
\begin{aligned}
v_{r} & =1-J i_{2 r-1,1}(a t)-J i_{2 r-1,3}(a t)+(2 r-1) J i_{2 r-1}^{(3)}(a t) \\
I_{r} & =(C / L)^{1 / 2}\left\{J i_{2 r, 1}(a t)+J i_{2 r, 3}(a t)-2 r J i_{2 r}^{(3)}(a t)\right\} \\
I_{0} & =(C / L)^{1 / 2}\left\{J i_{0.1}(a t)+J i_{0.3}(a t)-\frac{1}{2} a^{2} t^{2}\right\} \\
& =\frac{1}{2}(C / L)^{1 / 2}\left\{\left(1+a^{2} t^{2}\right) J i_{0.1}(a t)-a^{2} t^{2}\left(1+J_{1}(a t)\right)+a t J_{0}(a t)\right\} .
\end{aligned}
$$

where (21) follows from (20) by integration by parts.

If the line is discharged into inductance $\frac{1}{2} L$ and resistance,$(L / C)$ in series, the solution follows from (14) in place of (12).

\title{
ON CERTAIN INTEGRALS IN THE THEORY OF HEAT CONDUCTION*
}

By STEWART P.TTERSON (I.C.I. (Explosives) Limited, Sterenston, Scotland:

In a recent note ${ }^{1} \mathrm{~W}$. Horenstein evaluates the integrals

$$
\begin{aligned}
& \phi \equiv \int_{0}^{t} x^{-3 / 2} \exp \left(-\frac{a^{2}}{x}-b^{2} x\right) d x, \\
& \psi \equiv \int_{0}^{t} x^{-1 / 2} \exp \left(-\frac{a^{2}}{x}-b^{2} x\right) d x,
\end{aligned}
$$

in terms of the tabulated exponential and error functions. The evaluation of the more general integral, viz.

$$
\int_{1}^{1} \exp \left(-s^{2}-n^{2} / s^{2}\right) d s
$$

from which $\phi$ and $\psi$ are easily derived, was given by Riemann."

Integrals of the above type arise in the solution by classical methods of various heat conduction problems. It is the purpose of this note to point out that treatment of many such problems by the Heaviside "operational" or equivalent Laplace transform method leads directly and naturally to the required solution in tabulated functions.

Thus, to take a simple case, the classical solution of

$$
\frac{\partial \theta}{\partial t}=\frac{1}{4} \frac{\partial^{2} \theta}{\partial a^{2}}-b^{2} \theta ; \quad \theta \rightarrow 0, t \rightarrow 0, \theta \rightarrow 1, a \rightarrow 0+,
$$

* Received Nov. 24, 1945.

1 W. Horenstein, Quart. Appl. Math. 3, 183-184 (1945).

2 B. Riemann, Partielle Differentialgleichunien, 2nd ed., 1376, p. 173. 
(where $\theta$ is a function of $a$ and $t$ ) will be

$$
\theta=a \pi^{-1 / 2} \phi .
$$

If, however,

$$
\bar{\theta}(a, p) \equiv \int_{0}^{\infty} e^{-p t} \theta(a, t) d t
$$

equations (3) transform into

$$
\frac{\partial^{2} \bar{\theta}}{\partial a^{2}}=4\left(p+b^{2}\right) \bar{\theta} ; \quad \bar{\theta} \rightarrow \frac{1}{p}, a \rightarrow 0+
$$

which lead at once to

$$
\bar{\theta}=p^{-1} \exp \left[-2 a \sqrt{\left(p+b^{2}\right)}\right] .
$$

The inversion theorem for the Laplace transform then gives

$$
\theta=\frac{1}{2 \pi i} \int_{\gamma-i \infty}^{\gamma+i \infty} \lambda^{-1} \exp \left[\lambda t-2 a \sqrt{\left(\lambda+b^{2}\right)}\right] d \lambda,
$$

along the usual contour.

By a series of obvious and natural steps, ${ }^{3}$ it is easy to show that this is equal to

$$
\begin{aligned}
\frac{e^{2 a b}}{4 \pi i} \int_{\gamma^{\prime}-i \infty}^{\gamma^{\prime}+i \infty} \lambda^{-1} \exp [\lambda t & -2(a+b t) \sqrt{\lambda}] d \lambda+\frac{e^{-2 a b}}{4 \pi i} \int_{\gamma^{\prime \prime}-i \infty}^{\gamma^{\prime \prime}+i \infty} \lambda^{-1} \exp [\lambda t-2(a-b t) \sqrt{\lambda}] d \lambda, \\
= & \frac{e^{2 a b}}{2}\left[1-\operatorname{erf}\left(\frac{a}{\sqrt{ } \hat{t}}+b \sqrt{t}\right)\right]+\frac{e^{-2 a b}}{2}\left[1-\operatorname{erf}\left(\frac{a}{\sqrt{ } \bar{t}}-b \sqrt{ } t\right)\right],
\end{aligned}
$$

and it can be verified that this satisfies (3).

\section{NOTE ON A FORMULA FOR THE SOLUTION OF AN ARBITRARY ANALYTIC EQUATION*}

By HERBERT E. SALZER (Mathematical Tables Projec!, Nere York City)

In a recent note $\mathrm{D}$. $\mathrm{R}$. Blaskett and $\mathrm{H}$. Schwerdtfeger ${ }^{-1}$ give a fairly well known expansion for a root $\alpha$ of the equation $f(z)=0$, as a power series in $f\left(z_{0}\right)$, where $z_{0}$ is near $\alpha$, namely,

$$
\alpha=\sum_{\nu=0}^{\infty} \frac{(-1)^{v}\left[f\left(z_{0}\right)\right]^{\nu}}{\nu !}\left[\frac{d^{v} f^{-1}(w)}{d w^{\nu}}\right]_{v=r\left(z_{0}\right)} .
$$

where $w$ denotes $f(z)$.

Of use in connection with (1) is a paper by Van Orstrand, "Reversion of Power Series," Phil. Mag., (6) 19, 366-376 (1910). Van Orstrand's article deals with the re-

3 H. Jeffreys, Operational methods in mathematical physics, Cambridge, 1931, p. 70.

* Received January 26, 1946.

1 This Quarterly 3, 266-268 (1945) 